Ferrata Storti Foundation

\title{
Phase II-like murine trial identifies synergy between dexamethasone and dasatinib in T-cell acute lymphoblastic leukemia
}

Haematologica 2021

Volume 106(4):1056-1066

\section{Correspondence:}

FREDERIK W. VAN DELFT

frederik.van-delft@newcastle.ac.uk

Received: October 21, 2019.

Accepted: March 4, 2020.

Pre-published: March 5, 2020.

https://doi.org/10.3324/haematol.2019.241026

(C)2021 Ferrata Storti Foundation

Material published in Haematologica is covered by copyright. All rights are reserved to the Ferrata Storti Foundation. Use of published material is allowed under the following terms and conditions:

https://creativecommons.org/licenses/by-nc/4.0/legalcode. Copies of published material are allowed for personal or internal use. Sharing published material for non-commercial purposes is subject to the following conditions:

https://creativecommons.org/licenses/by-nc/4.0/legalcode, sect. 3. Reproducing and sharing published material for commercial purposes is not allowed without permission in writing from the publisher.

\author{
Yuzhe Shi, ${ }^{1}$ Melanie C. Beckett, ${ }^{1}$ Helen J. Blair, ${ }^{1}$ Ricky Tirtakusuma, ${ }^{1}$ \\ Sirintra Nakjang, ${ }^{1}$ Amir Enshaei, ${ }^{1}$ Christina Halsey, ${ }^{2}$ Josef Vormoor, ${ }^{3}$ \\ Olaf Heidenreich, ${ }^{1,3}$ Anja Krippner-Heidenreich ${ }^{3 \#}$ and Frederik W. van Delft ${ }^{1 *}$
}

${ }^{1}$ Wolfson Childhood Cancer Research Centre, Northern Institute for Cancer Research, Newcastle University, Newcastle upon Tyne, UK; ${ }^{2}$ Wolfson Wohl Cancer Research Centre, Institute of Cancer Sciences, College of Medical, Veterinary and Life Sciences, University of Glasgow, Glasgow, UK and ${ }^{3}$ Prinses Máxima Centrum voor Kinderoncologie, Utrecht, the Netherlands

${ }^{\#} A K H$ and FWvD contributed equally as co-senior authors.

\section{ABSTRACT}

-cell acute lymphoblastic leukemia (T-ALL) is frequently characterized by glucocorticoid (GC) resistance, which is associated with inferior outcomes, thus highlighting the need for novel therapeutic approaches for GC-resistant T-ALL. The pre-T-cell receptor (pTCR)/TCR signaling pathways play a critical role in cell fate decisions during physiological thymocyte development, with an interplay between TCR and glucocorticoid receptor (GR) signaling determining the T-lymphocyte selection process. We performed an shRNA screen in vitro and in vivo in T-ALL cell lines and patient-derived xenograft (PDX) samples to identify vulnerabilities in the pTCR/TCR pathway and identified a critical role for the lymphocyte cell-specific kinase (LCK) in cell proliferation. LCK knockdown or inhibition with dasatinib (DAS) caused cell cycle arrest. Combination of DAS with dexamethasone (DEX) resulted in significant drug synergy leading to cell death. The efficacy of this drug combination was underscored in a randomized phase II-like murine trial, recapitulating an early phase human clinical trial. T-ALL expansion in immunocompromised mice was significantly impaired using this drug combination, compared to mice receiving control vehicle or single drug treatment, highlighting the immediate clinical relevance of this drug combination for high-risk T-ALL patients. Our results thus provide a strategy to improve the efficacy of current chemotherapy platforms and circumvent GC resistance.

\section{Introduction}

Current minimal residual disease (MRD)-stratified chemotherapy protocols for patients with T-cell acute lymphoblastic leukemia (T-ALL) result in 5-year eventfree survival rates of $80 \%$ and $50 \%$ for pediatric and adult patients, respectively. ${ }^{1,2}$ Induction failure, early relapse, and isolated central nervous system (CNS) involvement are more common in T- than B-lineage ALL. ${ }^{3}$ Moreover, resistance to conventional chemotherapy including glucocorticoid (GC) is a frequent feature of relapsed and refractory T-ALL, reducing the second remission rate and long-term outcomes. ${ }^{4}$ GC are an instrumental component of ALL therapy and induce apoptosis in lymphoid malignancies. ${ }^{5.7}$ Resistance to GC is a critical factor influencing treatment response and outcome. ${ }^{5,8-11}$ Amongst ALL subtypes, GC resistance is more frequently observed in infant ALL and T-ALL. $5,9,11,12$

Endogenous GC can induce apoptosis during the selection process of $\mathrm{T}$ lymphocytes in the thymus, an effect which can be constrained by crosstalk with T-cell receptor (TCR) signaling. ${ }^{13,14}$ Whilst mature T-cell maintenance requires tonic TCR signaling, inappropriate TCR expression has been shown to give rise to T-cell malignancies in mouse model systems. ${ }^{15,16}$

The immature pre-T-cell receptor (pTCR) consists of a complex of alpha (pTCR $\alpha)$

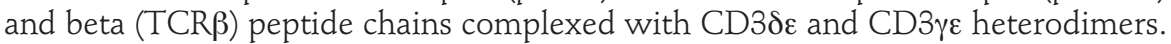


Activation of this complex occurs through the SRC family kinase (SFK) members lymphocyte cell-specific proteintyrosine kinase (LCK) and FYN. They are critical modulators of T-cell development and activation. ${ }^{17}$ LCK phosphorylates the plasma membrane-associated TCR complex ${ }^{18}$ and ZAP70 ${ }^{19}$ ZAP70 in turn phosphorylates the linker for activation of $T$ cells (LAT) leading to the activation of downstream signaling cascades. The overall activity of LCK is regulated by the phosphorylation status of the activating and inhibitory tyrosine residues 394 and 505, respectively. ${ }^{20}$ LCK activation correlates with Y416SRC (also Y394LCK) phosphorylation, as the latter over-rides the inhibitory effects of Y505 phosphorylation. ${ }^{21}$

We hypothesized, therefore, that T-ALL continues to rely on proliferative and survival stimuli inherent to the TCR signaling pathway, which, if inhibited, may enhance GC sensitivity. A targeted shRNA screen directed against components of the TCR signaling initiation complex identified a crucial role for LCK in T-ALL proliferation, both in vitro and in vivo. The anti-proliferative effects of LCK knockdown could be replicated by using the small molecule inhibitor dasatinib (DAS). Drug synergy was observed using DAS in combination with dexamethasone (DEX) on patient-derived xenograft (PDX) cell survival in vitro. Mirroring the design of early phase human trials, a murine phase II-like trial demonstrated significantly impaired leukemia progression in vivo using combination treatment. Our results present a clear rationale for using DAS in conjunction with DEX to enhance conventional chemotherapeutic treatment and revert GC resistance in pediatric T-ALL patients.

\section{Methods}

\section{Patient samples}

The patient-derived material was collected as part of diagnostic investigations of patients at the Great North Children's Hospital, Department of Paediatric Haematology and Oncology, Newcastle upon Tyne, UK. The material was collected and stored with informed consent obtained from all subjects in accordance with the Declaration of Helsinki.

Samples with explicit written consent for in vivo studies were requested from the Newcastle Biomedicine Biobank, Newcastle University, UK, and used according to approvals given by the Newcastle Biomedicine Biobank (NHB application NHB-008) and the local institutional review board Newcastle \& North Tyneside Ethics Committee (REC reference: 07/H0906/109).

\section{Drug matrix assays}

Dasatinib (9 nM - $30 \mu \mathrm{M})$ (DC Chemicals, Shanghai, China) was titrated on T-ALL cell lines $\left(4 \times 10^{4} /\right.$ well) in 96 -well plates (Corning, NY, USA). Cell viability was assessed after 3 days using Cell Counting Kit 8 (NBS Biologicals, Cambridgeshire, UK). Absorbance was measured at OD450 $\mathrm{nm}$ using a POLARstar Omega plate reader (BMG LABTECH, Bucks, UK). IC I0 $_{0}$ values were determined by GraphPad Prism. Assays were performed in triplicate and at least three independent repeats were performed.

For DAS/DEX combination treatments DAS (80 nM - 50 $\mu \mathrm{M})$ and DEX $(0.09 \mathrm{nM}-600 \mathrm{nM})$ were titrated in 2-dimensions on T-ALL cell lines ( $4 \times 10^{4}$ cells per well in 96-well plate) or ex vivo expanded PDX cells $\left(8 \times 10^{4}\right.$ cells per well in 96 -well plate). Ex vivo expansion was achieved after co-culture with OP9-DL1 for 1 week, after which cells were separated from their feeders by repetitive transfer and subsequently plated. After 72 hours (h) of culture, the plates were developed as above. Drug synergy was determined using Combenefit software (v.2.021). ${ }^{22}$

\section{Phase II-like murine trial}

For each of the ten PDX samples, $8 \times 10^{6}$ cells were intrafemorally (IF) injected into four NSG mice (40 mice in total) under isoflurane anesthesia. The four NSG mice derived from one PDX sample were matched for gender and age. T-ALL engraftment in mouse peripheral blood was monitored weekly by tail vein bleeds (20 $\mu \mathrm{L} \mathrm{blood/mouse).} \mathrm{The} \mathrm{four} \mathrm{mice} \mathrm{of} \mathrm{each} \mathrm{PDX} \mathrm{were} \mathrm{random-}$ ized to receive control vehicle, DAS (35 mg/kg), DEX (1 mg/ $/ \mathrm{kg}$ ) or DEX/DAS combination by intraperitoneal (IP) injection upon engraftment, defined as $\geq 0.5 \%$ peripheral blood $\mathrm{hCD} 45^{+} / \mathrm{hCD} 7^{+}$ cells. The median treatment duration of these mice was 15 days, depending on their clinical status. When any of the four mice displayed signs of ill health or weight loss, all four mice derived from this PDX were killed at the same time to assess leukemia engraftment in bone marrow, blood, spleen, liver and CNS. Spleen size and weight were recorded. Statistical analyses were performed using RStudio (Boston, MA, USA) with linear mix model. The final analysis excluded the four mice derived from patient sample LK214, as all mice succumbed to T-ALL before treatment was initiated.

See the Online Supplementary Appendix for further details of the methods used.

\section{Results}

\section{A targeted shRNA screen of T-cell receptor pathway components identifies an essential role for lymphocyte cell-specific kinase in T-cell acute lymphoblastic leukemia cell line and patient-derived xenograft proliferation in vitro}

To explore the importance of the pTCR/TCR signaling complex in proliferation and survival of malignant T cells, we performed a limited shRNA screen targeting six genes with three shRNAs per gene, including $L C K, Z A P 70$, PTCRA, FYN, CD3E and LAT in four T-ALL cell lines (HPBALL, CUTLL1, MOLT4, SUPT1), and included 18 control shRNAs (see Online Supplementary Methods and Online Supplementary Table S1A). In silico analysis using the Cancer Cell Line Encyclopedia (CCLE) demonstrated that these six genes are highly expressed in a panel of T-ALL cell lines (Online Supplementary Figure S1A). LCK and PTCRA expression was confirmed by targeted gene expression analysis in T-ALL cell lines and patient samples (Figure 1B) (Online Supplementary Figure S1B). The limited shRNA screen revealed the shLCK\#3 construct targeting $L C K$ was the only construct significantly depleted in all four cell lines, when compared with base line shRNA integration, underlining an important role for LCK in T-ALL cell line proliferation and/or survival (Figure 1A and Online Supplementary Figure S1C and D). The shLCK\#1, shZAP70\#1 and shPTCRA\#2 constructs were lost in 3 out of 4 cell lines. Constructs targeting FYN, CD $3 E$, or $L A T$ were significantly depleted in one cell line only, suggesting that these molecules do not play an universal role in T-ALL cell proliferation. ShRNAs against essential ribosomal genes were predictably depleted, whilst all three shRNA constructs targeting the tumor suppressor PTEN were enriched as expected. Repeated sampling at 16, 30 and 40 days after transduction demonstrated progressive depletion of shRNA constructs targeting LCK and ZAP70 (Online Supplementary Table S2 and Online Supplementary Figure S1E). PDX LK203 showed 
A
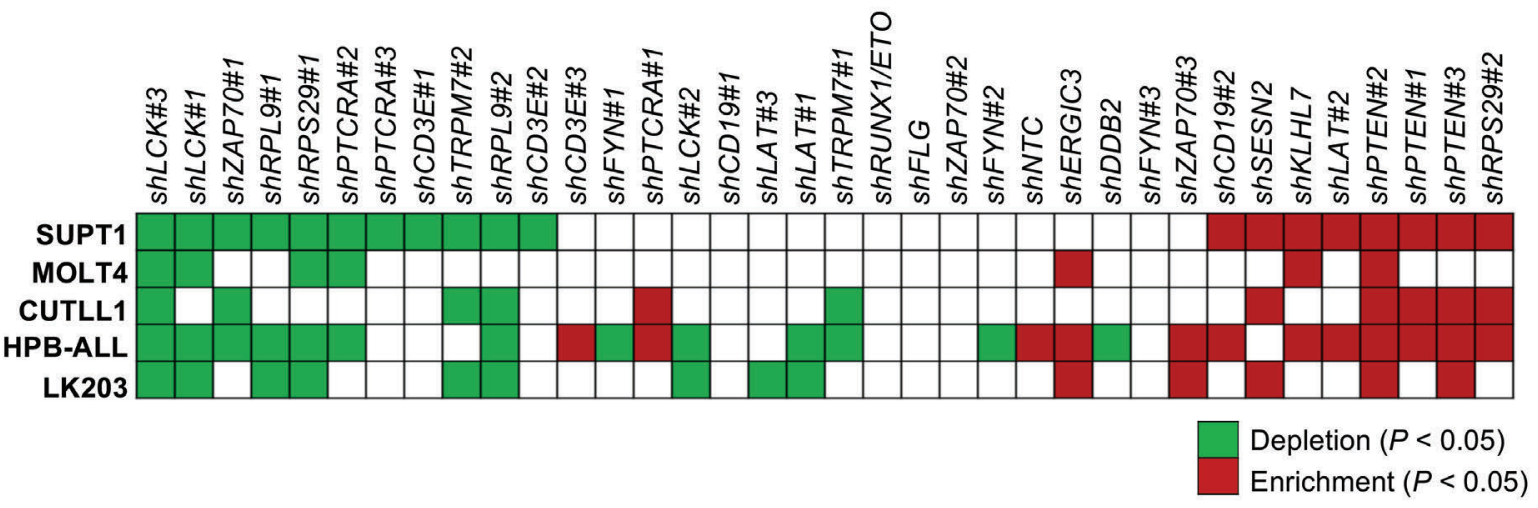

B

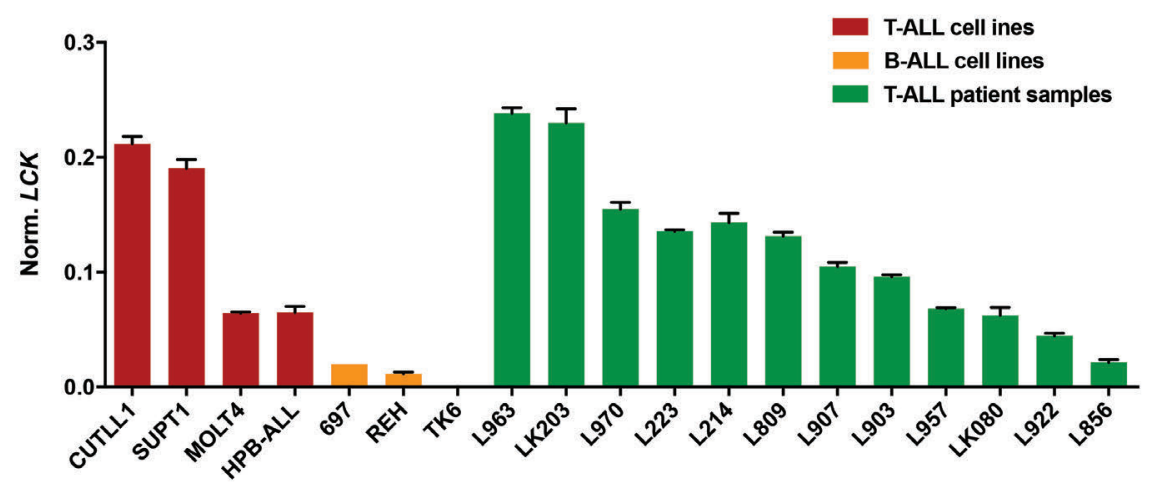

Figure 1. A targeted shRNA screen reveals lymphocyte cell-specific kinase (LCK) is essential for in vitro proliferative potential. (A) T-cell acute lymphoblastic leukemia (T-ALL) cell lines (SUPT1, MOLT4, HBP-ALL, CUTLL1) and PDX LK203 were subjected to a functional screen using a pLKO5-shRNA library containing 36 constructs targeting selected pre-T-cell receptor (pTCR)/TCR signaling complex components (PTCRA, CD3E, FYN, ZAP70, LCK, LAT), positive (PTEN, RPS29, RPL9), negative controls (KLHL7, CD19, DDB2, ERGIC3, FLG, RUNX1-ETO, SESN2, TRPM7) and a non-targeting control (NTC). Genomic DNA was sampled and barcoded. Enriched and depleted shRNAs were identified by next generation sequencing (NGS). The heatmap depicts statistically significant gains (red) or losses (green) of shRNA constructs after in vitro culture of four T-ALL cell lines (40 days) and PDX LK203 (30 days). (B) Relative gene expression of LCK in seven cell lines and 12 PDX samples. LCK expression was determined in four T-ALL cell lines, 697 and REH (B-lineage ALL cell lines [B-ALL]), TK6 (lymphoblastoid cell line), and 12 PDX samples by real time quantitative-polymerase chain reaction. GAPDH served as reference gene for normalization.

good viability $(\geq 75 \%)$ and proliferation potential $\left(\mathrm{T}_{\mathrm{d}}=2.8\right.$ days) in co-culture with human mesenchymal stem cells (hMSC), hence subjected to shRNA screening. ShRNA sequencing 30 days after transduction confirmed all constructs targeting LCK were significantly depleted (Figure 1A, Online Supplementary Table S3 and Online Supplementary Figure $S 1 F)$.

Knockdown of lymphocyte cell-specific kinase in T-cell acute lymphoblastic leukemia cell lines confirms an essential role for lymphocyte cell-specific kinase in vitro propagation

To confirm the role of LCK and other components of the pTCR/TCR signaling complex in cell proliferation, competitive outgrowth assays were performed. SUPT1, MOLT4 and CUTLL1 cells were transduced with lentiviral shRNAs targeting LCK, ZAP70, FYN, PTCRA or non-targeting control shRNAs. Successfully transduced cells expressing green fluorescence protein (GFP) were seeded in a 1:1 ratio with parental cells.

Three shRNAs were used to silence $L C K$, of which shLCK\#3 achieved the greatest degree of knockdown. Lentiviral knockdown with shLCK\#3 led to significant reduction in mRNA in SUPT1 (75\% KD), MOLT4 (55\% $\mathrm{KD})$, and CUTLL1 (45\% KD) cells (Figure 2A). In general, greater knockdown was associated with more pronounced impairment of in vitro proliferation (Online Supplementary Figure S2A). LCK expression was confirmed at protein level, demonstrating ubiquitous expression of LCK in cell lines (Online Supplementary Figure S1G). In line with mRNA downregulation, knockdown of LCK led to a decrease in total LCK protein expression (Figure 2A). Nontransduced cells consistently outcompeted LCK knockdown cells resulting in a pronounced loss of over $70 \%$ transduced $\mathrm{GFP}^{+}$cells in all three cell lines, underlining the critical and universal role of LCK in T-ALL cell line maintenance (Figure 2B and C, and Online Supplementary Figure $S 2 A$ and $C$ ).

A similar but less significant observation was made for ZAP70 knockdown in SUPT1, MOLT4 and CUTLL1 cells. Efficient ZAP70 knockdown correlated with a pronounced proliferation defect (Online Supplementary Figure S2A and $C)$. Knockdown of PTCRA affected proliferation in pTCR $\alpha^{+}$MOLT4 and SUPT1, but not in pTCR $\alpha$ - CUTLL1 (Online Supplementary Figure S2A and C). Moreover, FYN knockdown did not affect proliferation in any of the cell lines despite efficient knockdown (Online Supplementary Figure S2B).

Knockdown of lymphocyte cell-specific kinase in T-cell acute lymphoblastic leukemia cell lines and patient-derived xenograft samples impairs leukemia propagation in vivo

To confirm a functional role for LCK in vivo, PDX L963 cells were transduced with our shRNA library and transplanted into six NSG mice (Figure $3 \mathrm{~A}$ and Online 
A
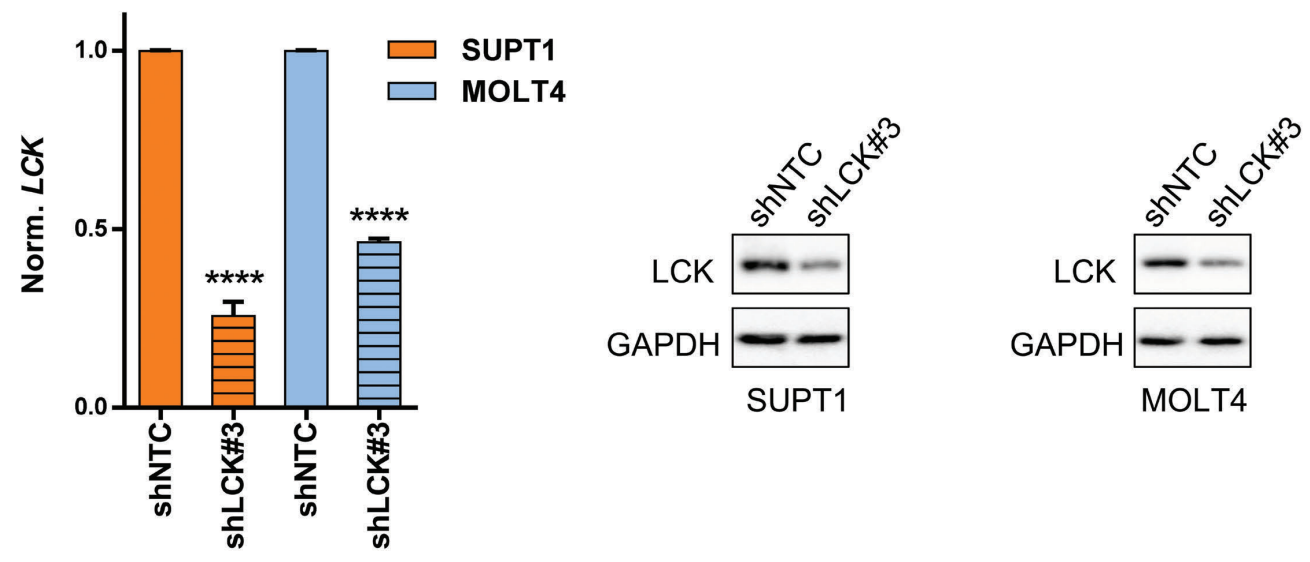

B

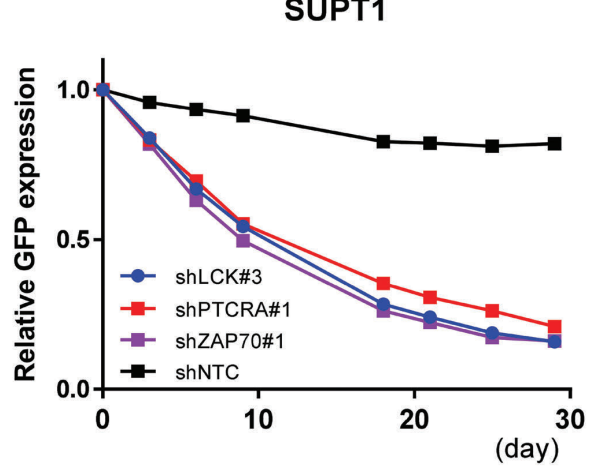

C

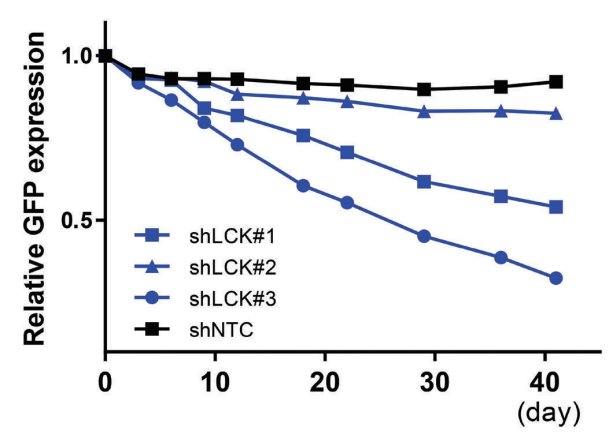

Figure 2. Knockdown of lymphocyte cell-specific kinase (LCK) reduces propagation of T-cell acute lymphoblastic leukemia (T-ALL) cell lines in vitro. SUPT1 (A and B) and MOLT4 (A and C) cells were lentivirally transduced with shNTC (non-targeting control), shLCK\#1, shLCK\#2, shLCK\#3, shPTCRA\#1, or shZAP70\#1 expression constructs. (A) Knockdown efficiency of LCK at mRNA level (left) and protein level (right) after 6 days. Whole cell lysates were probed for total LCK and GAPDH in western blot analysis. (B and C) T-ALL cell lines SUPT1 (B) and MOLT4 (C) transduced with GFP-expressing shLCK (blue), shZAP70 (purple), shPTCRA (red) or shNTC (black) constructs were seeded in a 1:1 ratio with non-transduced parental cells in vitro. Cells were cultured and analyzed repetitively by flow cytometry for the presence of GFP ${ }^{+}$cells over a time period of 30 and 40 days for SUPT1 and MOLT4, respectively. A relative GFP expression of 1 denotes a mixture of $50 \%$ GFP ${ }^{+}$cells with $50 \%$ parental cells (ratio 1:1). A value of 0.5 refers to $25 \%$ of GFP ${ }^{+}$cells and $75 \%$ parental cells (ratio $1: 4$ ). Student's $t$-test: $* * * * P<0.001$.

Supplementary Figure S3A). Genomic DNA (gDNA) was extracted from $\mathrm{L} 963$ cells isolated from bone marrow and spleen after mice became symptomatic (week 11). ShRNA sequencing indicated that shLCK\#3 represented the most significantly depleted shRNA construct in vivo (Figure 3A, Online Supplementary Table S3 and Online Supplementary Figure $S 3 B$ ).

To assess the effect of $L C K$ knockdown on engraftment fitness, MOLT4 cells were transduced with lentiviral vectors encoding either red fluorescent protein RFP/shNTC (non-targeting control) or GFP/shLCK\#3. Equal proportions of cell populations were transplanted into NSG mice $(n=5)$. Leukemia cells were isolated from spleen, bone marrow and liver once mice were symptomatic (day 26). Flow cytometric analysis of the leukemic cell population established that cells carrying shNTC had a clear competitive engraftment advantage over cells with LCK knockdown in all mice tissues sampled (Figure $3 \mathrm{~B}$ and Online Supplementary Figure S3C).

Knockdown of lymphocyte cell-specific kinase leads to cell cycle arrest in T-cell acute lymphoblastic leukemia cell lines and patient-derived xenograft samples

Next we investigated the mechanisms underlying the defect in proliferation, survival and engraftment observed after LCK knockdown. Jurkat, MOLT4 and SUPT1 cells were transduced with shLCK\#1/\#3 and cell cycle analyses performed. In all cell lines, we observed significant cell cycle arrest with an increase in $G_{0} / G_{1}$ phase and decrease in $S$ phase after LCK knockdown (Figure 4A-C and Online Supplementary Figure S4A).

ShLCK\#3 led to decreased protein levels of total LCK and activated $\mathrm{p}-\mathrm{Y} 416^{\mathrm{SRC}}$ in cell lines, suggesting activation status of LCK is associated with cell cycle arrest. In PDX L963, LCK knockdown led to a $45 \%$ reduction in total LCK expression, as well as a $71 \%$ reduction in $\mathrm{p}-\mathrm{Y} 416^{\mathrm{SRC}}$ as assessed by Phosflow (Figure 4D). This knockdown resulted in a decrease in $\mathrm{S}$ phase over time compared to control (Online Supplementary Figure S4B). The proliferative behavior of PDX cells was analyzed after labeling with cell trace violet (CTV). PDX L963 cells were transduced with shRNA constructs targeting LCK or a non-targeting control (NTC) and co-cultured with OP9-DL1 feeder cells for 13 days. The $L C K$ knockdown cells showed restricted proliferation compared to the control cells (Figure 4E). Confirmatory siRNA knockdown of $L C K$ was undertaken in PDX samples LK203 and L963. Knockdown of total and activated LCK was confirmed by Phosflow. Cell cycle 
A

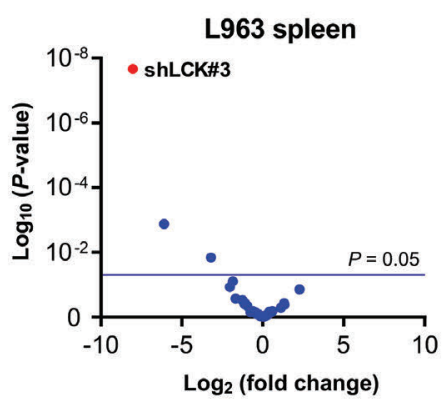

shLCK\#3

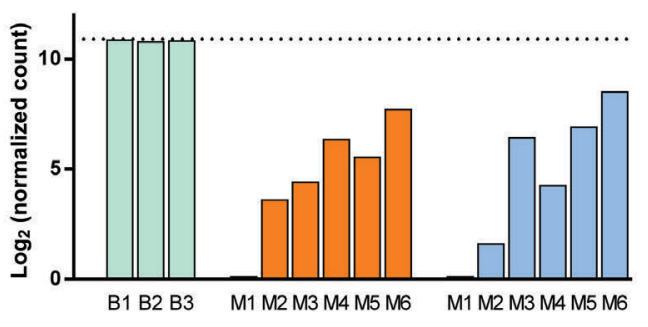

$\square$ Baseline

$\square$ Bone marrow

$\square$ Spleen

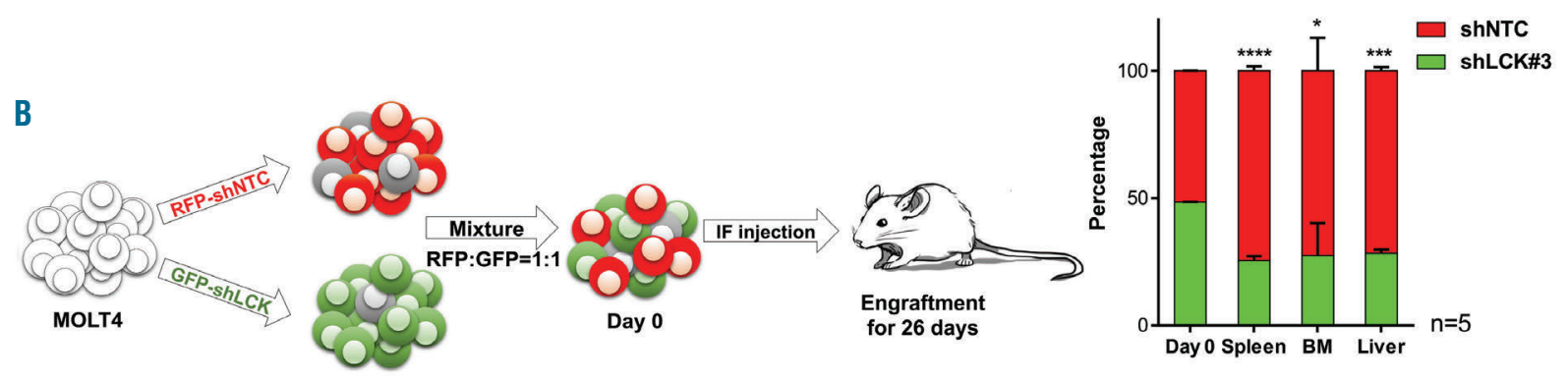

Figure 3. Loss of lymphocyte cell-specific kinase (LCK) negatively effects propagation potential of the T-cell acute lymphoblastic leukemia (T-ALL) cell line MOLT4 and patient-derived xenograft (PDX) L963 in vivo. (A) Volcano plot derived from the functional in vivo screen representing the magnitude of the fold change (log ${ }_{2}$ ) in shRNA abundance derived from leukemia cells isolated from the spleen of PDX L963 on the x-axis. Each dot represents an individual shRNA construct. The y-axis represents the significance in enrichment or depletion of shRNA constructs ( $\log _{10}$ scale). Three dots (shLCK\#3, shRPL9\#1 and shCD19\#2) above the blue line are significantly depleted $(P<0.05)$. Bar plot of the normalized shLCK\#3 sequencing reads $\left(\log _{2}\right)$ in leukemic cells derived from the bone marrow (orange) or spleen (blue) of six individual mice (M1-6), relative to the frequency of these reads before transplantation (green, base line B1-3). (B) Schematic representation of the in vivo competitive outgrowth assay. MOLT4 cells were lentivirally transduced with shNTC (red fluorescent protein, RFP) or shLCK\#3 (GFP) and intrafemorally injected into five NSG mice in a 1:1 ratio. Mice were culled once symptomatic and the ratio of RFP : GFP positive human leukemic cells in spleen ( $n=5$ ), bone marrow ( $n=3$ ) or liver $(n=3)$ determined by flow cytometry. In all mice, the MOLT4 cells carrying shLCK\#3 were outcompeted by shNTC cells during engraftment in spleen, marrow and liver Student's $t$-test: $* P<0.05, * * * P<0.005, * * * * P<0.001$.

arrest was observed, corroborating our earlier findings (Online Supplementary Figure S4C and D).

Knockdown of $L C K$ was analyzed for early apoptosis induction in CUTLL1, MOLT4, SUPT1 and Jurkat. Although a clear increase in Annexin V staining was observed in MOLT4, suggesting LCK knockdown led to apoptosis, this was not observed in CUTLL1, Jurkat or SUPT1 (Online Supplementary Figure S4E). This suggests that cell cycle arrest, rather than apoptosis induction, is the predominant effect leading to diminished cell expansion in vitro and reduced propagation in vivo after LCK knockdown.

Tyrosine kinase inhibitor dasatinib blocks lymphocyte cell-specific kinase function and leads to cell cycle arrest while lymphocyte cell-specific kinase activation levels predict response to its inhibition

The tyrosine kinase inhibitor DAS is a dual SRC/ABL inhibitor known to effectively inhibit LCK. ${ }^{21}$ The effect of DAS on LCK protein expression and activation status was assessed by western blot, after demonstrating near universal LCK activation as evidenced by tyrosine residue 416 phosphorylation in cell lines (Online Supplementary Figure $S 1 G)$. We confirmed that DAS effectively abolished activated $p-Y 416^{\text {SRC }}$ in all four T-ALL cell lines tested, whilst slightly decreasing total LCK protein levels. Furthermore, dephosphorylation of inhibitory $\mathrm{p}-\mathrm{Y} 505^{\mathrm{LCK}}$ was noted, as well as a decrease in $p-Y 783^{\text {PLCY1 }}$ and $p-Y 493^{\text {ZAP70 }}$, two downstream targets of LCK (Figure 5A and Online Supplementary Figure S5A). As knockdown of LCK leads to cell cycle arrest, we performed cell cycle analyses after administration of DAS. Cell cycle arrest was observed in all six T-ALL cell lines tested, with a significant increase in $\mathrm{G}_{0} / \mathrm{G}_{1}$ and decrease in $\mathrm{S}$ phase (Figure $5 \mathrm{~A}$ and Online Supplementary Figure S5B). In parallel, PDX cells supported by in vitro co-culture with OP9-DL1 were exposed to DAS In line with our cell line data, DAS abolished activated p-Y416 ${ }^{\text {SRC }}$ levels in all six PDX samples (Figure 5B and Online Supplementary Figure S5C) and cell cycle arrest was observed in all three PDX samples tested (Figure 5B and Online Supplementary Figure S5D).

The in vitro sensitivity of a panel of nine T-ALL cell lines to DAS was determined. The $\mathrm{IC}_{50}$ observed ranged from 5 $\mathrm{nM}$ (HSB2) to $15 \mu \mathrm{M}$ (MOLT16) (Figure 5C). The cell line HSB2 not only demonstrated the highest sensitivity to DAS but also the highest $\mathrm{p}-\mathrm{Y} 416^{\mathrm{SRC}}$ activation level as determined by Phosflow. This observation can be explained by the presence of a $\mathrm{t}(1 ; 7)(\mathrm{p} 34 ; \mathrm{q} 34)$ translocation leading to LCK activation by TCR $\beta$ enhancer elements in HSB2. We thus hypothesized that the level of activated LCK might represent a biomarker for DAS responsiveness. Phosflow was used to quantify and calcu-

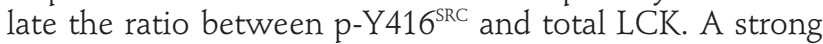
and significant correlation was observed between the $\mathrm{IC}_{50}$ for DAS and the ratio of activated Y416 $6^{\text {SRC }}$ in T-ALL cell lines $\left(\mathrm{R}_{2}=0.778, P=0.004\right)$ (Figure $5 \mathrm{C}$ ). The sensitivity of PDX cells to DAS ranged from $\mathrm{GI}_{50}$ of $23.8 \mathrm{nM}$ to $19.7 \mu \mathrm{M}$ (median of $1.2 \mu \mathrm{M}$ ). However, in this setting, no significant correlation between the $\mathrm{GI}_{50}$ and $\mathrm{p}-\mathrm{Y} 416^{\mathrm{SRC}} / \mathrm{LCK}$ ratio was identified, suggesting that DAS sensitivity of patientderived cells is dependent on additional factors (Online Supplementary Figure S5E). 
A
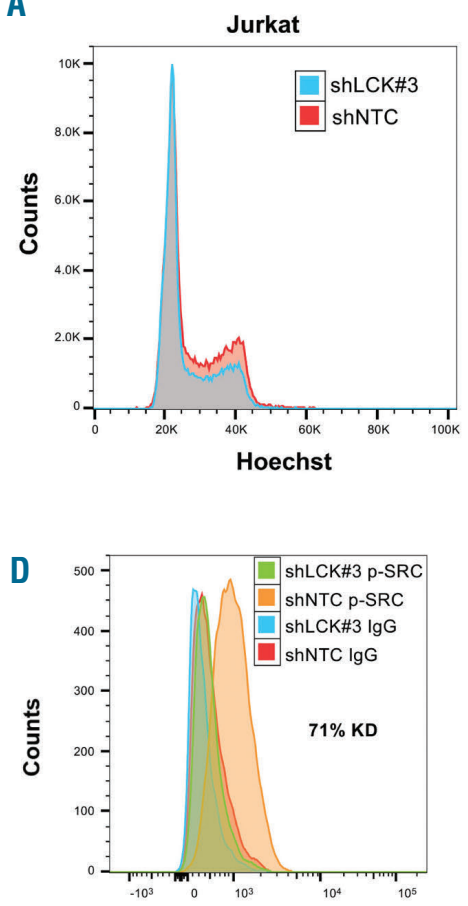

p-Y416 SRC
B

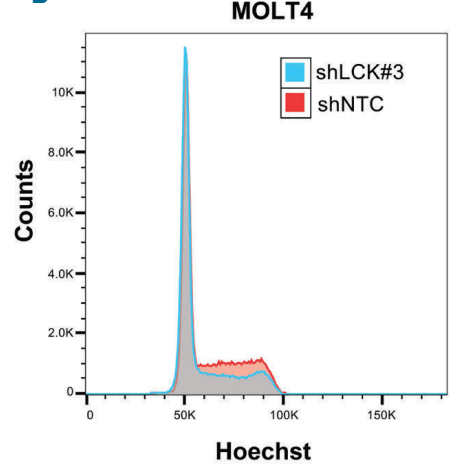

L963

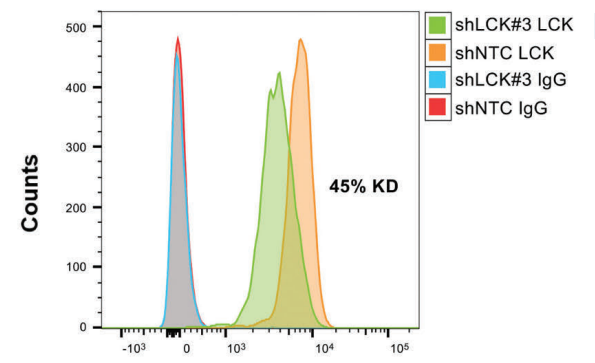

LCK
C

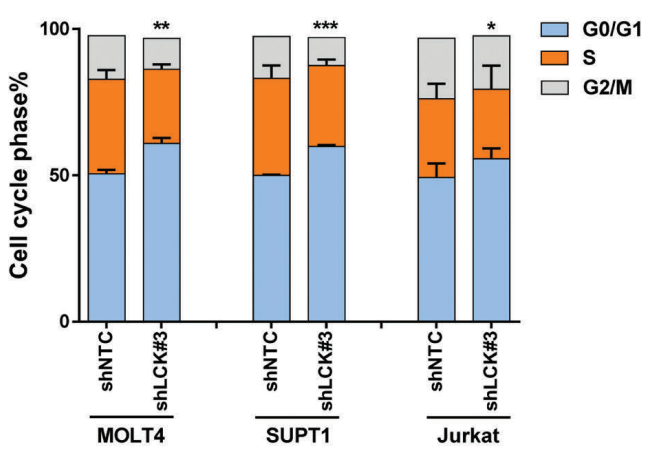

Figure 4. Lymphocyte cell-specific kinase (LCK) knockdown leads to cell cycle arrest in T-cell acute lymphoblastic leukemia (T-ALL) cell lines and patient-derived xenograft (PDX) cells. (A-C) Cell cycle status was determined by flow cytometry using Hoechst 33342 in cell lines Jurkat (A and C), MOLT4 (B and C), and SUPT1 (C) 7 days after transduction with shLCK\#3 or shNTC expression vectors. (D) PDX L963 cells were lentivirally transduced with shLCK\#3 or shNTC expression constructs. Phosflow analysis of total LCK and p-Y416 ${ }^{\mathrm{SRC}}$ was performed 8 days later. (E) shLCK\#3 and shNTC transduced PDX L963 cells were loaded with CTV and cultured for 13 days. Flow cytometric analysis of CTV incorporation (cell divisions) was performed and demonstrated progressive reduction in cell number after 4-6 cell divisions after LCK knockdown relative to control knockdown. Student's $t$-test: $* P<0.05, * * P<0.01, * * * P<0.005$.

Dasatinib re-sensitizes dexamethasone resistance in T-cell acute lymphoblastic leukemia cell lines and patient-derived xenograft samples

DAS leads to complete inhibition of $p-Y 416^{\text {SRC }}$ and cell cycle arrest in T-ALL cell lines and PDX cells, suggesting that DAS treatment of T-ALL has a cytostatic effect. In clinical practice, effective eradication of T-ALL relies on the application of combinatorial treatment. LCK inhibition has previously been shown to sensitize chronic lymphoid leukemia (CLL) to DEX and induce cell death. ${ }^{23}$ We thus went on to investigate potential synergy between LCK inhibition and DEX, as DEX is universally used for treatment of ALL. The cell viability of SUPT1 and CUTLL1, in the presence of DEX, was evaluated after knockdown of LCK. Whereas the cell viability of mock transduced and non-targeting control cells was minimally affected by DEX treatment, LCK knockdown increased DEX sensitivity suggesting that LCK protein and/or activity levels play a crucial role in GC resistance (Figure 6A and Online Supplementary Figure S6A).

A more detailed analysis of the potency of the combination of DEX and LCK inhibition was examined by using DAS instead of the LCK knockdown. DEX (0-600 nM) and DAS (0-50 $\mu \mathrm{M})$ were titrated along a dose matrix and cell viability was determined. Synergy for individual drug combinations was determined using Combenefit. ${ }^{22}$ The matrix revealed drug synergy at concentrations which are clinically achieved, i.e., $100 \mathrm{nM}$ for DEX and $264 \mathrm{nM}$ for DAS (Figure $6 \mathrm{~B}$ and Online Supplementary Figure S6B). ${ }^{24,25}$ Bioinformatic analysis of all ten T-ALL cell lines revealed a statistically significant enrichment of drug synergy at clin- ically relevant concentrations. This synergy was observed at 8-110 nM of DEX and 0.223-4.5 $\mathrm{MM}$ of DAS (Online Supplementary Figure S6C and D).

Subsequently, PDX cells were expanded ex vivo for 1 week and exposed to the same drug combinations in dose matrices. These assays verified the synergistic action of $\mathrm{DEX}+\mathrm{DAS}$ in a wide range of PDX cells, whilst confirming that increased DAS concentrations and resultant LCK inhibition augmented the response to DEX (Figure 6C and Online Supplementary Figure S6E). Combined analysis of all drug matrices with PDX cells again revealed a statistically significant enrichment of drug synergy at clinically relevant concentrations (Online Supplementary Figure S6F). Moreover, the combination of DEX+DAS induced more cell death compared with control vehicle or single drugs as revealed by Annexin V/PI staining (Figure 6C).

DEX has a wide range of actions, including genomic and non-genomic effects. Genomic effects are the result of nuclear translocation of the GC receptor and subsequent transactivation or repression of genes containing a GC response element (GRE), as exemplified by the Glucocorticoid-Induced Leucine Zipper (GILZ) gene. Accordingly, we observed strong induction of GILZ gene expression after DEX exposure in the T-ALL cell line Jurkat and five PDX samples tested (Figure 6D and Online Supplementary Figure S6G). This response was significantly enhanced when combining DEX with knockdown of $L C K$ (Figure $6 \mathrm{D}$ ) or DEX+DAS in a range of T-ALL cell lines and PDX samples, suggesting that LCK inactivation augments DEX-induced gene transcription and reverses DEX resistance (Figure 6D and Online Supplementary Figure S6G). 


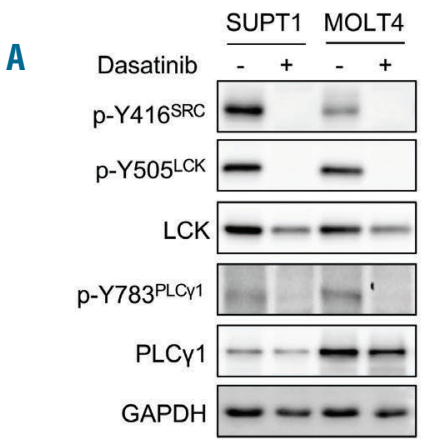

B

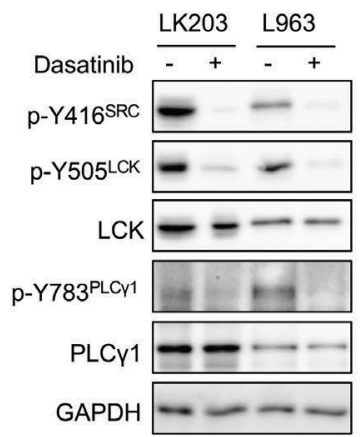

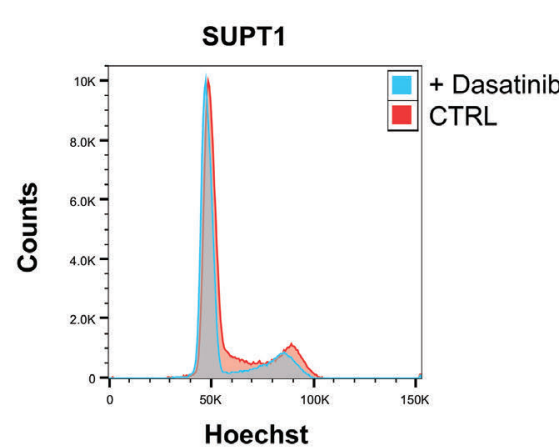
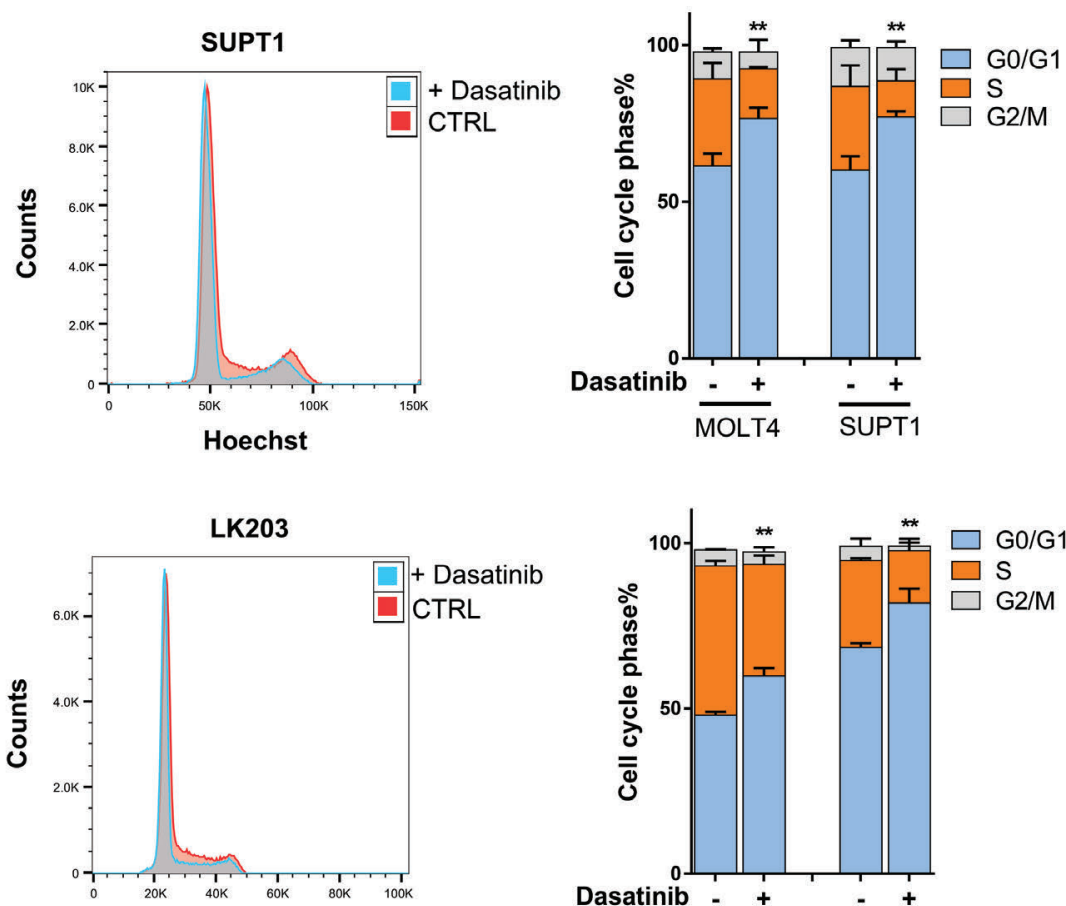

Hoechst

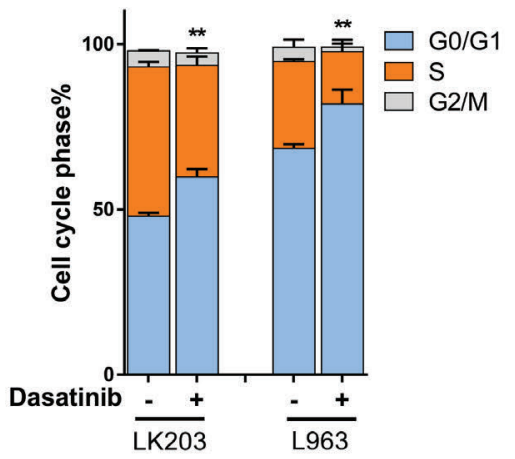

C

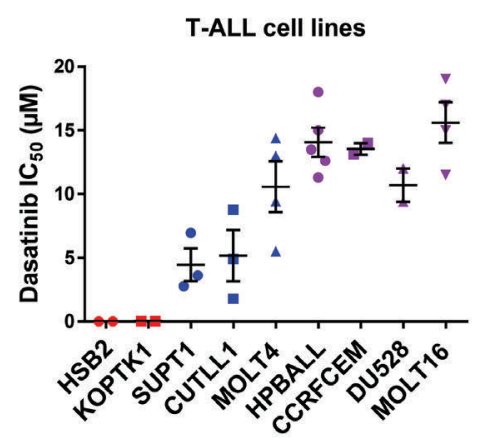

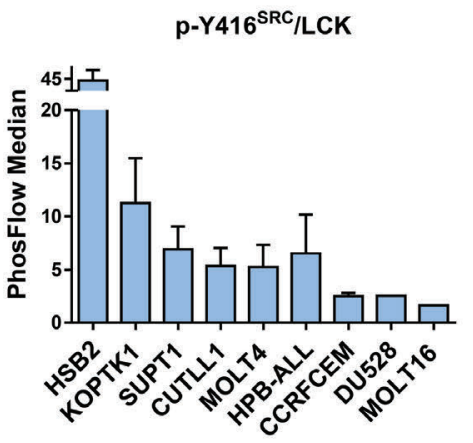

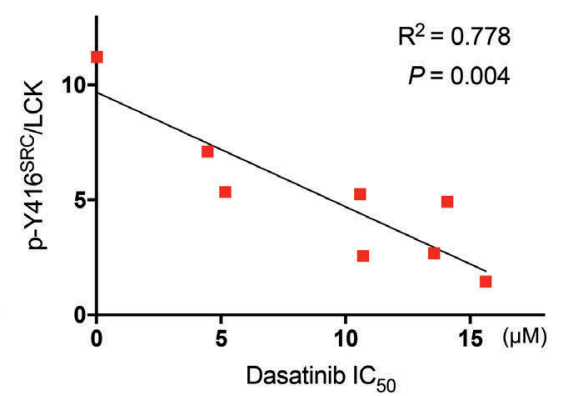

Figure 5. Dasatinib (DAS) inhibits lymphocyte cell-specific kinase (LCK) and leads to cell cycle arrest. (A) SUPT1 and MOLT4 cells were treated with vehicle control or DAS $(2 \mu \mathrm{M})$ for 24 hours $(\mathrm{h})$ and expression of total LCK, activated p-Y416 ${ }^{\mathrm{SRC}}$, total PLCy1 and activated p-Y783 $3^{\mathrm{PLC} y}$ was assessed in western blot analysis (left) or cells were stained with Hoechst and analyzed for cell cycle status (middle and right). (B) Patient-derived xenograft (PDX) LK203 and L963 cells were treated for 24 $\mathrm{h}$ with $1 \mu \mathrm{M}$ of DAS. LCK and PLC $\gamma 1$ total protein and activating phospho-sites at Y416 and Y783 were assessed in western blot analyses or PDX were stained with

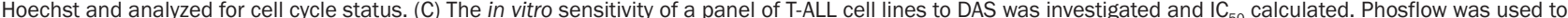
determine the ratio of activated $\mathrm{p}-\mathrm{Y} 416^{\mathrm{SRC}} /$ total $\mathrm{LCK}$. This ratio was correlated with in vitro sensitivity to DAS $\left(\mathrm{R}^{2}=0.778, P=0.004\right)$. Student's $t$-test: $* \star P<0.01$. NB HSB2 was excluded from this analysis, as the extreme sensitivity to DAS was caused by the presence of a unique translocation absent in all other sensitive cell lines.

Phase II-like trial in vivo demonstrates significant reduction in leukemia burden after combination treatment with dexamethasone and dasatinib

To test the efficacy of DEX and DAS in vivo, we conducted a phase II-like trial in mice (Figure 7A). ${ }^{26}$ Ten PDXs were engrafted in four mice each. The four mice derived from one single patient sample were randomly assigned to treatment arms, namely control vehicle, DEX $(1 \mathrm{mg} / \mathrm{kg})$, DAS $(35 \mathrm{mg} / \mathrm{kg})$ or DEX+DAS $(1 \mathrm{mg} / \mathrm{kg}$ DEX $+35 \mathrm{mg} / \mathrm{kg}$ DAS). After IF injection, mice tail vein blood was monitored weekly for human CD7/CD45 and murine CD45 expression to monitor peripheral blood engraftment. Representative PDX L809 commenced treatment 46 days after injection for a total duration of 3 weeks; the four mice were culled 72 days after injection (Figure 7B). L809 cells engrafted in the spleens of the four mice showed greatly reduced levels of total LCK and dephosphorylation of LCK ( $p-Y 416^{\text {SRC }}$ and $\left.p-Y 505^{\mathrm{LCK}}\right)$ after DAS or DEX+DAS combination treatment (Figure 7C). Western blot analysis of positively selected viable human cells again demonstrated decreased protein expression of LCK and $\mathrm{p}-\mathrm{Y} 416^{\mathrm{SRC}}$ after DAS treatment. The number of residual viable human cells after effective DEX+DAS treatment was not sufficient to categorically confirm reduced protein expression (Online Supplementary Figure S7F). One mouse in the DAS arm (LK080) developed uterine prolapse before dosing commenced and the mice derived from PDX LK214 succumbed during the first week of treatment. These five were excluded from the final analysis. Combining the results of 35 mice derived from nine patient samples, DEX+DAS treatment significantly impaired leukemia progression more than single drug DEX, DAS or control vehi- 
A

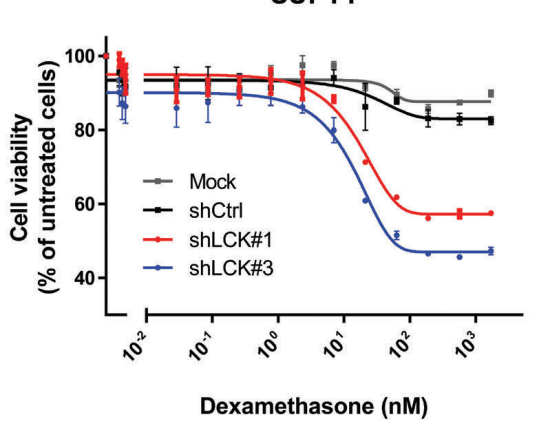

C

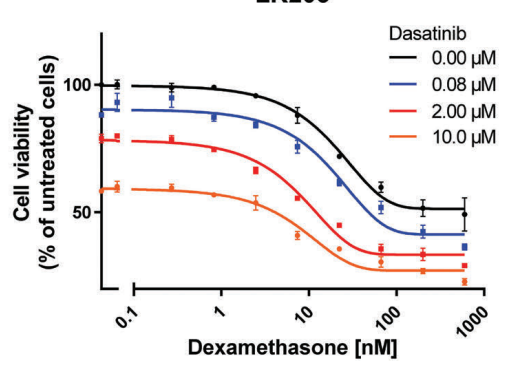

B

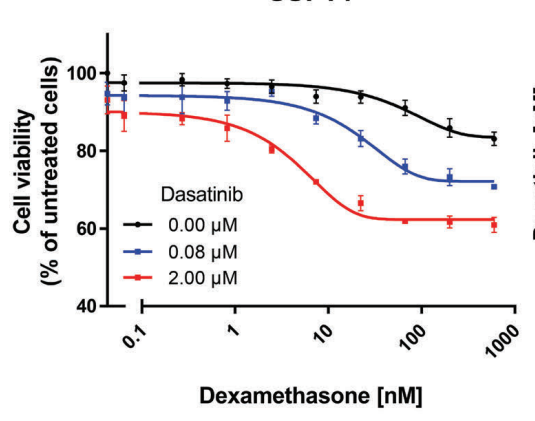

Dexamethasone [nM]

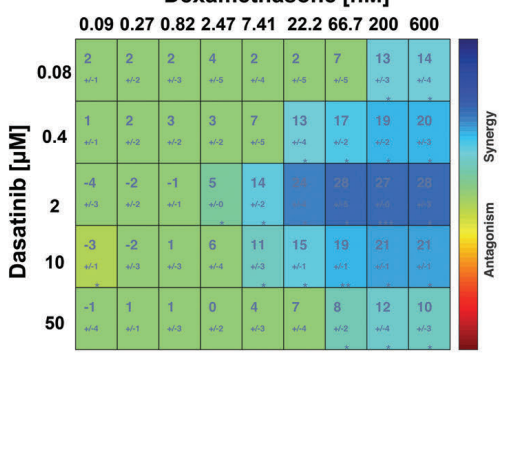

LK203

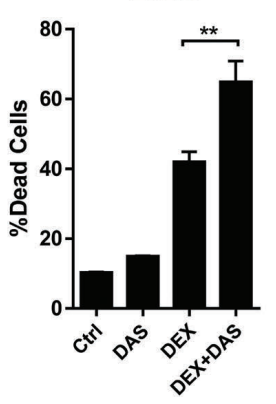

D

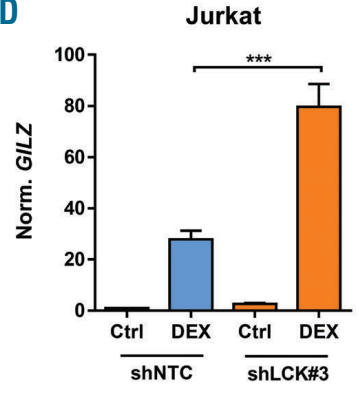

Jurkat

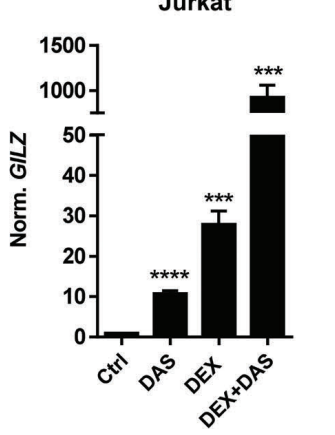

Figure 6. Dexamethasone (DEX) and dasatinib (DAS) act synergistically to induce cell death in T-cell acute lymphoblastic leukemia (T-ALL). (A) Cell viability of parental SUPT1 cells (mock), shCtrl (NTC), shLCK\#1 or shLCK\#3 transduced SUPT1 cells upon treatment with increasing DEX concentrations (0-1699 nM). (B) Cell viability of SUPT1 with and without DAS (left; black line, no DAS; blue line, $0.8 \mu \mathrm{M}$; red line, $2.0 \mu \mathrm{M}$ ) in combination with increasing concentrations of DEX (0-600 $\mathrm{nM})$ as derived from the drug matrix with titration of DEX $(0-600 \mathrm{nM})$ and DAS $(0-50 \mathrm{uM}$; right). (Right) Combenefit analysis of drug matrix demonstrates drug synergy in SUPT1 cells at clinically relevant drug concentrations. (C) (Left) LK203 cells were expanded ex vivo on OP9-DL1 feeder cells for 1 week prior to treatment with and without DAS (black line, no DAS; blue line, $0.08 \mu \mathrm{M}$; red line, $2.0 \mu \mathrm{M}$; orange line, $10 \mu \mathrm{M}$ ) in combination with increasing concentrations of DEX (0-600 nM) as derived from a drug matrix with DEX (0-600 nM) and DAS (0 - $50 \mathrm{uM})$ (Online Supplementary Figure S6E). (Right) Cell death analysis in LK203 cells exposed to control (Ctrl) conditions, DAS $(1 \mu \mathrm{M})$, DEX (100 nM) or DAS+DEX combination treatment. (D) (Left) Normalized GILZ mRNA expression in Jurkat cells after transduction with shNTC or shLCK\#3 with or without DEX exposure (100 nM). (Right) Normalized GILZ mRNA expression in Jurkat cells after exposure to Ctrl conditions, DAS $(2 \mu \mathrm{M})$, DEX $(100 \mathrm{nM})$ or DAS+DEX combination treatment at the same concentrations. Student's $t$-test: $* * P<0.01, * * * P<0.005, * * * * P<0.001$.

cle as measured by hCD45 or hCD7 engraftment in peripheral blood, bone marrow, spleen and CNS (Figure 7D-F and Online Supplementary Figure S7A). Single agent DEX reduced CNS leukemia burden in 7 out of 9 samples, reflecting its proven efficacy in reducing CNS relapses, ${ }^{27}$ whilst DAS showed some reduction in CNS burden in 4 out of 8 samples (Online Supplementary Figure S7B). Combination therapy was particularly effective, with complete eradication of measurable CNS leukemia in five patient samples and evidence of an additive effect with DEX in 3 out of 4 of the remaining patient samples (Online Supplementary Figure S7B). When considering the cohort of nine patient samples overall, combination treatment significantly reduced leukemic infiltrates compared to control $(P=0.02)$. Representative histology images are shown in Figure 7G. Spleen weight was substantially reduced in mice receiving combination treatment, compared with the single or control treatment arms (Online Supplementary Figure $S 7 C$ and D). DEX+DAS also significantly reduced $\mathrm{hCD} 45^{+}$or $\mathrm{hCD}^{+}$leukemia cell engraftment in liver tissue of all six PDX samples analyzed (Online Supplementary Figure STE). The initial therapeutic advantage of DEX+DAS in mice derived from PDX LK080 was lost at the end of the experiment, most likely because these mice were kept alive for 3 weeks after completion of treatment. In the case of L907, however, the benefit of combination treatment was not observed until the last time point (Online Supplementary Figure S7G).

\section{Discussion}

Using a phase II-like murine trial, we demonstrate here the efficacy of the drug combination DEX+DAS in impairing expansion of human T-ALL samples. This effect is apparent in an unselected, biologically heterogeneous, cohort of PDX samples. This trial format recapitulates early phase human clinical trials and indicates that this drug combination could be widely applicable in the treatment of T-ALL. Studies by Serafin et al. first proposed a role for this drug combination. ${ }^{28}$ Our murine trial extends these initial observations with an extensive cohort consisting of nine different PDX demonstrating treatment advantage for both DEX sensitive and resistant T-ALL.

Significant superiority of DEX+DAS was demonstrated even after exclusion of mice who reached their clinical end points prematurely. These untoward events highlight the practicalities of performing murine trials.

We propose that the impaired in vivo expansion results from a combination of cell cycle arrest as well as cell death. Several mechanisms could provide plausible explanations for the occurrence of cell cycle arrest. DAS is a protein tyrosine kinase inhibitor which targets $\mathrm{Abl}$ and SFK family members. We have confirmed that DAS effectively inhibits activity of the SFK member LCK by preventing phosphorylation, leading to $G_{0} / G_{1}$ arrest. DAS has previously been shown to inhibit cyclin dependent kinase 1 (CDK1), which plays a central role in G1/S and G2/M 

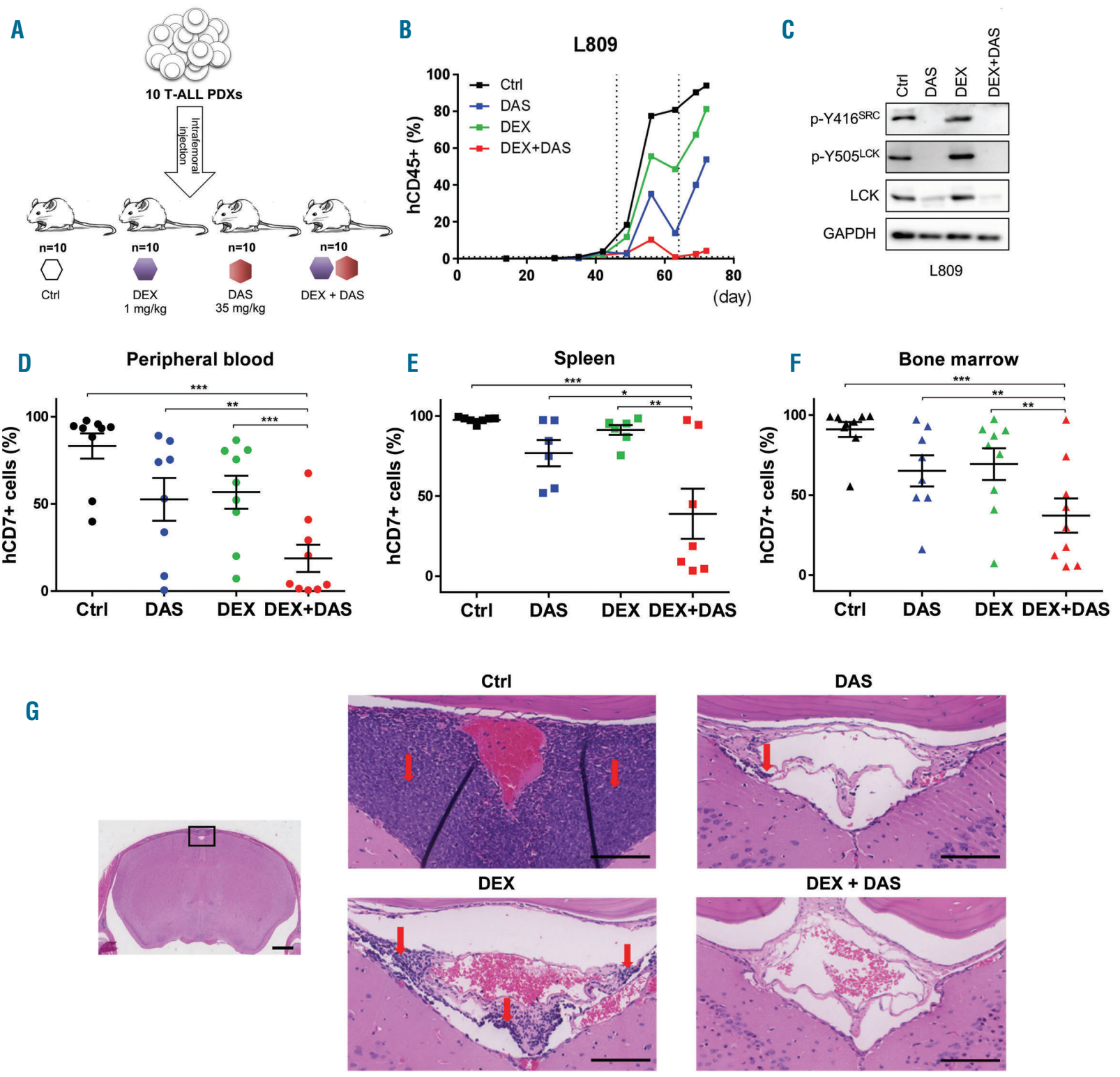

Figure 7. Dexamethasone (DEX) + dasatinib (DAS) synergize to impair leukemia engraftment in a Phase Il-like murine trial. (A) Layout of the in vivo trial using ten different patient-derived xenograft (PDX) samples. PDX samples were engrafted into four mice each and treated with control vehicle (Ctrl), DEX (1 mg/kg), DAS (35 $\mathrm{mg} / \mathrm{kg}$ ) or DEX+DAS (1 mg/kg DEX + $35 \mathrm{mg} / \mathrm{kg}$ DAS). Mice were dosed once daily, 5 times per week, for $2-3$ weeks depending on clinical status of the mice. (B) Engraftment of hCD45 $5^{+}$cells (\%) was determined weekly in peripheral blood derived from four mice injected with PDX L809. Engraftment levels are shown starting from day of injection (day 0) in mice receiving control vehicle (Ctrl, black), DAS (blue), DEX (green), or DEX+DAS (red). Vertical dotted lines indicate the treatment window (3 weeks) starting on day 46 and completing on day 64. Mice were culled on day 72 and analyzed for hCD45/hCD7 engraftment. (C) Western blotting of total and phosphorylated lymphocyte cell-specific kinase (LCK) protein levels of whole cell lysates derived from the spleens of four mice injected with PDX L809 under four different treatment arms (Ctrl, DAS, DEX or DEX+DAS) relative to the housekeeper GAPDH. (D-F) Summary of final human CD7 ${ }^{+}$engraftment (\%) in peripheral blood (D), spleen $(E)$, and bone marrow (F) of mice treated with Ctrl (black), DAS (blue), DEX (green), or DEX+DAS (red). $* P<0.05, * \star P<0.01, * * * P<0.001 .(G)$ Photomicrographs of whole brain-skull sections stained with Hematoxylin \& Eosin from PDX L809. (Left) Low power scout view of whole brain with area shown in al other images marked by black box. (Centre and right) High power view (x20 objective) of meninges around the central venous sinus in mice receiving Ctrl, DAS, DEX or DEX+DAS treatment. Red arrows mark the leukemic infiltrate. Scale bar marks $1 \mathrm{~mm}$ on scout view and $100 \mu \mathrm{m}$ on high power images.

transition. $^{29}$ Furthermore, G1 cell cycle arrest, through upregulation of the cyclin-dependent kinase inhibitors p21CIP1 (CDKN1A) and p27KIP1 (CDKN1B), has been observed after DAS treatment in acute myeloid leukemia. ${ }^{30}$ We propose that LCK is the predominant target of DAS in this disease setting, as our shRNA screen identified a critical role for LCK in cell proliferation in cell lines and PDX samples. Moreover, LCK is the proposed DAS target when blocking T-cell activation. ${ }^{21}$ Competitive assays confirmed defective proliferation of T-ALL cells after LCK knockdown in vitro and in vivo. We have shown that LCK knockdown leads to $G_{0} / G_{1}$ cell cycle arrest in cell lines and PDX. This effect was more pronounced using DAS, a finding which could potentially be explained by incomplete knockdown of LCK or the wide spectrum of kinases targeted by DAS.

As reported earlier and confirmed in our studies, DAS is cytotoxic to a small subset of T-ALL samples with $\mathrm{IC}_{50}$ values in the low nanomolar range. ${ }^{11}$ These observations were made in T-ALL samples without kinase activating mutations, which are seen very infrequently in T-ALL. To the best of our knowledge, our cohort includes only one 
PDX with such an activating genetic lesion (LK287, FIP1L1-PDGFRA). Cytotoxicity to DAS is significantly increased upon combination with DEX. Our data indicate drug synergy between DAS and DEX at clinically relevant concentrations. A previous, mostly in vitro, study advocated the use of DEX+DAS in GC resistant T-ALL. ${ }^{28}$ Our extended studies indicate DEX+DAS act synergistically in the majority of cell lines and PDX tested independent of their prior sensitivity to DEX. The potential of DEX+DAS to revert $\mathrm{GC}$ resistance is an exciting observation. GC resistance is frequently observed in relapsed/refractory $\mathrm{T}$-ALL, ${ }^{4}$ and DEX+DAS provide a clinically actionable approach to re-sensitize T-ALL resistant to DEX.

The implementation of DAS into clinical management would benefit from the identification of a reliable response biomarker. Although LCK activation status (ratio p-Y416 SRC/LCK) strongly correlates with DAS sensitivity in cell lines, we were unable to corroborate this observation in PDX cells. Sample size and intricacies of in vitro assays using PDX cells could provide possible explanations for these inconsistencies. Nevertheless, in vivo drug synergy was observed in the majority of samples tested. Of interest, drug response profiling of T-ALL samples suggested SRC pathway activation may represent a response biomarker. $^{31}$

The mechanism underlying the observed drug synergy remains to be fully elucidated. T-cell activation can be blocked by using clinically relevant concentrations of the tyrosine kinase inhibitor DAS, which binds to the ATPbinding pocket of LCK thereby preventing the phosphorylation of the activating loop of the kinase domain $\mathrm{p}-Y 416^{\mathrm{SRC}}{ }^{21,32}$ When DEX is combined with DAS, physiological $\mathrm{CD}^{+} \mathrm{T}$-cell proliferation is reduced in an additive way. ${ }^{33,34}$ Furthermore, it has been previously suggested that the Calcineurin/NFAT/IL-4 axis is activated in patients exhibiting a prednisone poor response. ${ }^{28}$ We have shown here that combination of DEX+DAS significantly increases GILZ gene expression, reflecting increased transcriptional activity of the GC receptor. We thus hypothesize that inhibition of LCK disrupts the TCR-GR complex and established crosstalk between the TCR and GR path- ways leading to dissociation and transcriptional activation of the GR. ${ }^{13}$

To conclude, drug resistant T-ALL continues to represent an unmet clinical need. We provide further support for the inclusion of DAS in the treatment of T-ALL. It has been reported that DAS in combination with conventional chemotherapy is safe and well tolerated in children and young adults, although hematologic toxicity was significant. ${ }^{35}$ Thus, the DEX+DAS combination should be considered in the early phase setting to evaluate toxicity and efficacy in patients with GC resistant disease with or without cerebral spinal fluid involvement.

\section{Disclosures}

No conflicts of interest to disclose.

\section{Contributions}

$F W v D, A K H$ and $Y S$ designed the research; YS performed the research; $Y S, M C B, H J B, O H$ and $R T$ designed and performed the in vivo experiments; $F W v D, A K H$ and $Y S$ analyzed the data and wrote the paper; $S N$ and $A E$ performed the bioinformatics analysis; $\mathrm{CH}$ performed brain histology and imaging; JV, OH and $\mathrm{CH}$ reviewed the manuscript.

\section{Acknowledgments}

The authors would like to thank patients, parents, and hospital staff at the Great North Children's Hospital, Newcastle upon Tyne, UK, for their valuable collaboration. The authors would like to thank Lynn Stevenson and Clare Orange, University of Glasgow, for brain histology and imaging. The brain histology slides were scanned by Glasgow University slide scanning and image analysis service at the Queen Elizabeth University Hospital, Glasgow. CH was funded by the Chief Scientist Office (ETM/374).

\section{Funding}

This work was supported by a Newcastle University Research Fellowship (to FWvD), Chinese Scholarship Council (CSC) (to YS), JGW Patterson Foundation (to MCB), North of England Children's Cancer Research, Action Medical Research (to FWvD).

\section{References}

1. Marks DI, Paietta EM, Moorman AV, et al. T-cell acute lymphoblastic leukemia in adults: clinical features, immunophenotype, cytogenetics, and outcome from the large randomized prospective trial (UKALL XII/ECOG 2993). Blood. 2009;114(25):51365145 .

2. Patrick K, Wade R, Goulden N, et al. Outcome for children and young people with early T-cell precursor acute lymphoblastic leukaemia treated on a contemporary protocol, UKALL 2003. Br J Haematol. 2014;166(3):421-424.

3. Goldberg JM, Silverman LB, Levy DE, et al. Childhood T-cell acute lymphoblastic leukemia: the Dana-Farber Cancer Institute acute lymphoblastic leukemia consortium experience. J Clin Oncol. 2003;21(19):36163622 .

4. Raetz EA, Borowitz MJ, Devidas M, et al. Reinduction platform for children with first marrow relapse of acute lymphoblastic leukemia: a Children's Oncology Group study[corrected]. J Clin Oncol. 2008;26(24):3971-3978.

5. Inaba H, Pui CH. Glucocorticoid use in acute lymphoblastic leukaemia. Lancet Oncol. 2010;11(11):1096-1106.

6. Pui CH, Carroll WL, Meshinchi S, Arceci RJ. Biology, risk stratification, and therapy of pediatric acute leukemias: an update. J Clin Oncol. 2011;29(5):551-565.

7. Pui CH, Mullighan CG, Evans WE, Relling MV. Pediatric acute lymphoblastic leukemia: where are we going and how do we get there? Blood. 2012;120(6):1165-1174.

8. Dordelmann M, Reiter A, Borkhardt A, et al. Prednisone response is the strongest predictor of treatment outcome in infant acute lymphoblastic leukemia. Blood. 1999; 94(4):1209-1217

9. Hongo I, Yajima S, Sakurai M, Horikoshi Y, Hanada R. In vitro drug sensitivity testing can predict induction failure and early relapse of childhood acute lymphoblastic leukemia. Blood. 1997;89(8):2959-2965

10. Kaspers GJ, Wijnands JJ, Hartmann R, et al. Immunophenotypic cell lineage and in vitro cellular drug resistance in childhood relapsed acute lymphoblastic leukaemia. Eur J Cancer. 2005;41(9):1300-1303.

11. Klumper E, Pieters R, Veerman AJ, et al. In vitro cellular drug resistance in children with relapsed/refractory acute lymphoblastic leukemia. Blood. 1995;86(10):3861-3868.

12. Pieters $\mathrm{R}$, den Boer ML, Durian $\mathrm{M}$, et al. Relation between age, immunophenotype and in vitro drug resistance in 395 children with acute lymphoblastic leukemia-implications for treatment of infants. Leukemia. 1998;12(9):1344-1348.

13. Jamieson CA, Yamamoto KR. Crosstalk pathway for inhibition of glucocorticoidinduced apoptosis by $\mathrm{T}$ cell receptor signaling. Proc Natl Acad Sci U S A. 2000; 97(13):7319-7324.

14. Ashwell JD, King LB, Vacchio MS. Crosstalk between the T cell antigen receptor and the glucocorticoid receptor regulates thymocyte development. Stem Cells. 1996;14(5):490-500.

15. Cui Y, Onozawa M, Garber HR, et al. Thymic expression of a T-cell receptor targeting a tumor-associated antigen coexpressed in the thymus induces T-ALL. 
Blood. 2015;125(19):2958-2967.

16. Serwold T, Hochedlinger K, Swindle J, Hedgpeth J, Jaenisch R, Weissman IL. T-cell receptor-driven lymphomagenesis in mice derived from a reprogrammed T cell. Proc Natl Acad Sci U S A. 2010;107(44):1893918943.

17. Palacios EH, Weiss A. Function of the Srcfamily kinases, Lck and Fyn, in T-cell development and activation. Oncogene. 2004:23(48):7990-8000

18. Iwashima M, Irving BA, van Oers NS, Chan AC, Weiss A. Sequential interactions of the TCR with two distinct cytoplasmic tyrosine kinases. Science. 1994;263(5150):1136-1139.

19. van Oers NS, Killeen N, Weiss A. Lck regulates the tyrosine phosphorylation of the $\mathrm{T}$ cell receptor subunits and ZAP-70 in murine thymocytes. J Exp Med. 1996;183(3):10531062.

20. Nyakeriga AM, Garg H, Joshi A. TCRinduced $\mathrm{T}$ cell activation leads to simultaneous phosphorylation at Y505 and Y394 of p56(lck) residues. Cytometry A. 2012;81(9):797-805.

21. Lee KC, Ouwehand I, Giannini AL, Thomas NS, Dibb NJ, Bijlmakers MJ. Lck is a key target of imatinib and dasatinib in T-cell activation. Leukemia. 2010;24(4):896-900.

22. Di Veroli GY, Fornari C, Wang D, et al. Combenefit: an interactive platform for the analysis and visualization of drug combinations. Bioinformatics. 2016;32(18):2866-2868.

23. Harr MW, Caimi PF, McColl KS, et al.
Inhibition of Lck enhances glucocorticoid sensitivity and apoptosis in lymphoid cell lines and in chronic lymphocytic leukemia. Cell Death Differ. 2010;17(9):1381-1391.

24. Liston DR, Davis M. Clinically relevant concentrations of anticancer drugs: a guide for nonclinical studies. Clin Cancer Res. 2017;23(14):3489-3498.

25. Yang L, Panetta JC, Cai X, et al. Asparaginase may influence dexamethasone pharmacokinetics in acute lymphoblastic leukemia. J Clin Oncol. 2008;26(12):19321939.

26. Townsend EC, Murakami MA, Christodoulou A, et al. The Public Repository of Xenografts enables discovery and randomized Phase II-like trials in mice. Cancer Cell. 2016;30(1):183.

27. Mitchell CD, Richards SM, Kinsey SE Lilleyman J, Vora A, Eden TO; Medical Research Council Childhood Leukaemia Working P. Benefit of dexamethasone compared with prednisolone for childhood acute lymphoblastic leukaemia: results of the UK Medical Research Council ALL97 randomized trial. Br J Haematol. 2005;129(6):734 745.

28. Serafin V, Capuzzo G, Milani G, et al Glucocorticoid resistance is reverted by LCK inhibition in pediatric T-cell acute lymphoblastic leukemia. Blood. 2017; 130(25):2750-2761.

29. Kruewel T, Schenone S, Radi M, et al. Molecular characterization of c-Abl/c-Src kinase inhibitors targeted against murine tumour progenitor cells that express stem cell markers. PLoS One. 2010;5(11):e14143.

30. Guerrouahen BS, Futami M, Vaklavas C, et al. Dasatinib inhibits the growth of molecularly heterogeneous myeloid leukemias. Clin Cancer Res. 2010;16(4):1149-1158.

31. Frismantas V, Dobay MP, Rinaldi A, et al. Ex vivo drug response profiling detects recurrent sensitivity patterns in drug-resistant acute lymphoblastic leukemia. Blood. 2017; 129(11):e26-e37.

32. Schade AE, Schieven GL, Townsend R, et al. Dasatinib, a small-molecule protein tyrosine kinase inhibitor, inhibits T-cell activation and proliferation. Blood. 2008;111(3):13661377.

33. Nerreter T, Distler E, Kochel C, Einsele H, Herr W, Seggewiss-Bernhardt R. Combining dasatinib with dexamethasone long-term leads to maintenance of antiviral and antileukemia specific cytotoxic $\mathrm{T}$ cell responses in vitro. Exp Hematol. 2013;41(7): 604-614.e4.

34. Smith LK, Cidlowski JA. Glucocorticoidinduced apoptosis of healthy and malignant lymphocytes. Prog Brain Res. 2010;182:1-30

35. Slayton WB, Schultz KR, Kairalla JA, et al. Dasatinib plus intensive chemotherapy in children, adolescents, and young adults with Philadelphia chromosome-positive acute lymphoblastic leukemia: results of Children's Oncology Group trial AALL0622. J Clin Oncol. 2018;36(22):2306-2314. 